\title{
Influence of mild-moderate hypocapnia on intracranial pressure slow waves activity in TBI
}

\author{
Erta Beqiri $^{1,2}$ - Marek Czosnyka ${ }^{1} \cdot$ Afroditi D. Lalou $^{1} \cdot$ Frederick A. Zeiler $^{3,4} \cdot$ Marta Fedriga $^{1,5} \cdot$ Luzius A. Steiner $^{6}$. \\ Arturo Chieregato $^{7} \cdot$ Peter Smielewski $^{1}$
}

Received: 20 July 2019 / Accepted: 23 October 2019 / Published online: 16 December 2019

(C) The Author(s) 2019

\begin{abstract}
Background In traumatic brain injury (TBI) the patterns of intracranial pressure (ICP) waveforms may reflect pathological processes that ultimately lead to unfavorable outcome. In particular, ICP slow waves (sw) $(0.005-0.05 \mathrm{~Hz})$ magnitude and complexity have been shown to have positive association with favorable outcome. Mild-moderate hypocapnia is currently used for short periods to treat critical elevations in ICP. Our goals were to assess changes in the ICP sw activity occurring following sudden onset of mild-moderate hypocapnia and to examine the relationship between changes in ICP sw activity and other physiological variables during the hypocapnic challenge.

Methods ICP, arterial blood pressure (ABP), and bilateral middle cerebral artery blood flow velocity (FV), were prospectively collected in 29 adult severe TBI patients requiring ICP monitoring and mechanical ventilation in whom a minute volume ventilation increase (15-20\% increase in respiratory minute volume) was performed as part of a clinical $\mathrm{CO}_{2}$-reactivity test. The time series were first treated using FFT filter (pass-band set to $0.005-0.05 \mathrm{~Hz}$ ). Power spectral density analysis was performed. We calculated the following: mean value, standard deviation, variance and coefficient of variation in the time domain; total power and frequency centroid in the frequency domain; cerebrospinal compliance $(\mathrm{Ci})$ and compensatory reserve index (RAP).

Results Hypocapnia led to a decrease in power and increase in frequency centroid and entropy of slow waves in ICP and FV (not ABP). In a multiple linear regression model, RAP at the baseline was the strongest predictor for the decrease in the power of ICP slow waves $(p<0.001)$.

Conclusion In severe TBI patients, a sudden mild-moderate hypocapnia induces a decrease in mean ICP and FV, but also in slow waves power of both signals. At the same time, it increases their higher frequency content and their morphological complexity. The difference in power of the ICP slow waves between the baseline and the hypocapnia period depends on the baseline cerebrospinal compensatory reserve as measured by RAP.
\end{abstract}

Keywords ICP slow waves $\cdot$ Hypocapnia $\cdot$ TBI $\cdot$ Cerebral autoregulation $\cdot$ Compensatory reserve $\cdot$ RAP

\section{Abbreviations and acronyms}

TBI Traumatic brain injury

This article is part of the Topical Collection on Brain trauma

Erta Beqiri

erta.beqiri@gmail.com

1 Brain Physics Laboratory, Division of Neurosurgery, Department of Clinical Neurosciences, University of Cambridge, Cambridge, UK

2 Department of Physiology and Transplantation, Milan University, Milan, Italy

3 Department of Surgery, Rady Faculty of Health Sciences, University of Manitoba, Manitoba, Canada
ICP Intracranial pressure

ABP Arterial blood pressure

4 Division of Anaesthesia, Department of Medicine, University of Cambridge, Cambridge, UK

5 Department of Anesthesia, Critical care and Emergency, Spedali Civili University Hospital, Piazzale Spedali civili 1, 25123 Brescia, Italy

6 Anesthesiology, University Hospital Basel and Department of Clinical Research University of Basel, Basel, Switzerland

7 Neurointensive Care Unit, Grande Ospedale Metropolitano Niguarda, Milan, Italy 


\begin{tabular}{|c|c|}
\hline FV & Flow velocity \\
\hline $\mathrm{Ci}$ & Cerebrospinal compliance \\
\hline FFT & Fast Fourier transform \\
\hline RAP & Compensatory reserve index \\
\hline $\mathrm{CBF}$ & Cerebral blood flow \\
\hline CBV & Cerebral blood volume \\
\hline MCA & Middle cerebral artery \\
\hline CPP & Cerebral perfusion pressure \\
\hline $\mathrm{PaCO} 2$ & Arterial partial pressure of carbon dioxide \\
\hline $\mathrm{pCO}_{2}$ & Partial pressure of carbon dioxide \\
\hline FVl & Flow velocity left \\
\hline $\mathrm{FVr}$ & Flow velocity right \\
\hline $\mathrm{SjvO}_{2}$ & Jugular bulb venous oxygen saturation \\
\hline sw & Slow wave \\
\hline RAP_FT & Fisher transformation of RAP \\
\hline PSD & Power spectral density \\
\hline ABP_sw & Slow wave component of ABP \\
\hline ICP_sw & Slow wave component of ICP \\
\hline FVl_sw & Slow wave component of FVl \\
\hline FVr_sw & Slow wave component of $\mathrm{FVr}$ \\
\hline CaBV & Cerebral arterial blood volume \\
\hline
\end{tabular}

\section{Introduction}

In traumatic brain injury (TBI) the mean value of intracranial pressure (ICP) might not be sufficient to help fully interpret the clinical status of the patient, while ICP waveforms contain information about the nature of the cerebrospinal circulation pathophysiology. ICP waveform can be decomposed into the following components defined in the frequency domain: the pulse waveform (which fundamental harmonic component frequency equals the heart rate), the respiratory waveform (related to the frequency of the respiratory cycle, 8-20 cycles/minute) and the "Slow waves" [7] which were previously defined in the Lundberg thesis as B waves with "frequency $0.5-2 / \mathrm{min}$ with amplitude from discernibility to $20 \mathrm{mmHg}$ " [24] and which definition was modified and adjusted in the latest years. Slow waves can be defined as oscillations in cerebral pressures and cerebral blood flow of duration longer than those of the respiratory origin with a spectral representation within the frequency limits defined roughly as 0.005 $0.05 \mathrm{~Hz}$ [7]. Analysis of slow waves in ICP could provide information about cerebral blood flow (CBF) autoregulation [36], brain compliance [27, 24] and brainstem activity [37, 22, 15]. Moreover, various parameters derived from slow waves, in particular higher magnitude [2] and higher complexity [23], were shown to be associated with outcome after TBI.

The vasogenic nature of ICP slow waves $[37,1]$ is underlined by the fact that rhythmic changes in cerebral blood volume (CBV) are transmitted into the ICP waveform [27]. Rhythmic oscillations in diameter of cerebral vessels can be triggered by fluctuations in mean arterial blood pressure
(ABP) and/or by local neurochemical mechanisms [20, 16]. These vascular changes are responsible for alterations in CBV and subsequently contribute to the oscillations observed in the cerebral blood flow velocity (FV) measured at the middle cerebral artery (MCA) [25]. CBV slow waves are ultimately transmitted into the ICP waveform [27]. The intracranial compliance modulates the transmission of the vasogenic waves. An increase in ICP slow wave amplitude may be indicative of an exhausted cerebrospinal compensatory reserve [39]. Slow wave magnitude has been shown to be suppressed by general anesthesia in awake versus sedated and ventilated TBI patients [21].

The intracranial blood pressure slow oscillations are likely modulated by the mean arterial blood pressure (ABP) and the sympathetic cervical system [17]. The relationship between ICP and ABP slow waves depends on the status of the dynamic autoregulation. With properly functioning $\mathrm{CBF}$ autoregulation, ICP slow waves are thought to result from the autoregulatory response to spontaneous fluctuations of cerebral perfusion pressure (CPP; where $\mathrm{CPP}=\mathrm{ABP}-\mathrm{ICP})[30$ ], and therefore can be used to gauge cerebral autoregulation quantitatively [9]. Failure of autoregulation modifies the relationship between ABP and ICP [36] in such a way that the response in ICP to the fluctuations in ABP becomes pressure passive and depends on the arterial bed compliance [14].

Changes in $\mathrm{PaCO} 2$ were suggested to be involved in the generation of the ICP slow waves, in the original Lundberg description of what he defined as B waves [24]. The arterial $\mathrm{pCO}_{2}$ fluctuations are not considered anymore the main generator of slow waves since they can be seen in ventilated patients, where $\mathrm{pCO}_{2}$ is actively stabilized and can be assumed to be kept constant. They are indeed considered potential modulators of the slow waves activity $[31,10]$ given their influence on the vascular resistance (metabolic regulation of $\mathrm{CBF}$ ). A decrease in $\mathrm{PaCO} 2$ (inducing brain alkalosis) produces, with intact vascular reactivity, an acute vasoconstriction which leads to a reduction of CBF and CBV and ultimately a decrease in ICP [4], as a function of intracranial compliance [40]. Prolonged prophylactic deep hyperventilation was used in the past to prevent ICP hypertension, but it is no longer recommended given the lack of positive association with outcome and harmful effect of lowering $\mathrm{CBF}$ in the most vulnerable areas to ischemic levels $[26,6]$. On the contrary, mild hypocapnia is considered safe from the hemodynamic point of view [32].

The behavior of ICP slow waves during hypocapnia in TBI patients has not been extensively studied yet, and it is uncertain whether there are significant changes in their activity and their patterns. Given that slow wave patterns are currently used to inform the treating clinicians about important physiological parameters (such as cerebral autoregulation, brain stem activity, and intracranial compliance) it is necessary to evaluate how a clinical intervention such as hypocapnia could influence the slow waves activity and therefore modify the assessment of the related physiology. 
Therefore, we conducted this retrospective study, bearing in mind two main objectives:

1. To assess changes in the ICP slow waves activity occurring following sudden onset of mild-moderate hypocapnia, using a variety of approaches, both in time and frequency domain.

2. To examine the relationship between changes in ICP slow wave activity and other physiological variables during the hypocapnia period.

\section{Materials and methods}

We present a retrospective analysis of waveform recordings of ICP, ABP, and bilateral MCA blood flow velocity (left - FVl, and right - $\mathrm{FVr}$ ), prospectively collected during $\mathrm{CO}_{2}$-reactivity studies in adult (age $>16$ years) severe TBI patients requiring ICP monitoring and mechanical ventilation admitted in the Neurocritical Care Unit (NCCU) at Addenbrooke's Hospital, Cambridge, from March 2001 to February 2002. Reaching back to digital recordings from past studies was motivated by new findings regarding slow ICP waves and the role of autoregulation assessment $[21,3]$.

As part of the clinical protocol on NCCU, all patients underwent routine testing of $\mathrm{CO}_{2}$-reactivity to aid prognostic stratification. The collection of these data was prospectively considered by the multidisciplinary NCCU Users Group, and it was agreed that because assessment of $\mathrm{CO}_{2}$-reactivity was part of normal clinical management and since no patient confidentiality issues were involved, formal informed consent was not required. Within our institution, patient data may be collected with waiver of formal consent, as long as it remains fully anonymized, with no method of tracing this back to an individual patient. This anonymous data is then provided for future research purposes. Such data curation remains within compliance for research integrity as outlined in the UK Health Departments (2011) Governance arrangements for research ethics committees.

Exclusion criteria included respiratory failure, a baseline pa $\mathrm{CO}_{2}<4.30 \mathrm{kPa}$, failure to obtain satisfactory bilateral transcranial Doppler signals and decompressive craniectomy. All patients were treated according to a CPP-orientated protocol aiming to keep CPP above $70 \mathrm{mmHg}$, ICP below $25 \mathrm{mmHg}$, and jugular bulb venous oxygen saturation $\left(\mathrm{SjvO}_{2}\right)$ above $50 \%$.

During the studies, all physiological parameters were maintained within the limits specified in the treatment guidelines of the unit. All patients were sedated with propofol $(2-5 \mathrm{mg} / \mathrm{kg} /$ $\mathrm{h}$ ) and fentanyl (1-2 mcg/kg/h), and paralyzed (atracurium). Infusion rates of sedative and vasoactive drugs were not changed and body temperature was kept constant throughout the study period.

\section{Data collection}

The data were collected as part of a prospective study [35]. The setting up can be briefly described as follows.

ICP monitoring was performed using an intraparenchymal probe (Codman MicroSensors ICP Transducer, Codman \& Shurtleff, Raynham, MA, USA). ABP was monitored invasively using a pressure monitoring kit (Baxter Healthcare CA, USA; Sidcup, UK) at the radial artery, zeroed at the level of the heart. Mainstream end-tidal $\mathrm{CO}_{2}$ monitoring was used (Marquette Solar $8000 \mathrm{M}$, GE Medical Systems, UK) to assess the stability of $\mathrm{CO}_{2}$ levels, but the related signal was not collected. FV was measured from the middle cerebral arteries (left and right) with two $2-\mathrm{MHz}$ probes with the Doppler Box (Multi Dop X4, DWL Elektronische Systeme, Sipplingen, Germany). The two probes were held in place with a Lam head rack. $\mathrm{SjO}_{2}$ was also monitored.

Data were collected during routine determination of $\mathrm{CO}_{2-}$ reactivity as part of the standard clinical practice on the unit. After recording baseline data for $20 \mathrm{~min}$ and obtaining a baseline value for $\mathrm{PaCO}_{2}$ (AVLOmni, Roche Diagnostics $\mathrm{GmbH}$, Graz, Austria), the minute volume of the ventilator was increased by a relative value of $15-20 \%$ of the original amount. If due to this intervention the unit's standard treatment guidelines $\left(\mathrm{PaCO}_{2}>3.5 \mathrm{kPa}\right.$ and or $\left.\mathrm{SjO}_{2}>55 \%\right)$ were exceeded, the protocol was abandoned. After an initial stabilization period of $10 \mathrm{~min}$, end-tidal $\mathrm{CO}_{2}$ was kept stable and data were recorded for further $20 \mathrm{~min}$. $\mathrm{PaCO}_{2}$ was measured at the middle of this stable phase ( 2 or 3 samples). During the study, all patients were sedated with propofol, $(2-5 \mathrm{mg} / \mathrm{kg} / \mathrm{h})$ and fentanyl (1-2 $\mathrm{mcg} / \mathrm{kg} / \mathrm{h}$ ), and paralyzed (atracurium). Infusion rates of sedative and vasoactive drugs were not changed and body temperature was kept constant throughout the study period. After $\mathrm{CO}_{2}$-reactivity testing had been completed, $\mathrm{PaCO}_{2}$ was slowly adjusted to the level that the responsible physician deemed appropriate.

The signals were acquired with a sampling frequency of $30 \mathrm{~Hz}$ using an analogue-digital converter (DT9801 and DT9803, Data Translation, Marlboro, MA, USA), and recorded using $\mathrm{ICM}+{ }^{\circledR}$ software (Cambridge Enterprise, Cambridge, UK, http://icmplus.neurosurg.cam.ac.uk).

\section{Data processing}

$\mathrm{ICM}+{ }^{\circledR}$ software (Cambridge Enterprise Ltd, Cambridge, UK, http://icmplus.neurosurg.cam.ac.uk) was used to process the recorded signals.

The artifacts were manually removed in the $30 \mathrm{~Hz}$ raw data: in the ABP signal, the arterial line flushes (corresponding to the arterial blood sampling) were removed; in ABP, ICP, 
$\mathrm{FVl}$, and FVr signal transient events, defined as short-lasting (less than $10 \mathrm{~min}$ ) events occurring less frequently than $3 / 10$ min, were removed to eliminate their influence on slow wave frequency bandwidth; any other artifacts found in recording were removed.

Per each patient recording, two periods-baseline and hypocapnic challenge - could be detected according to variations in the ICP trend (it decreases in hypocapnia; the initial transition and stabilization period after the increase in minute volume was excluded), the blood sampling time points (visible as arterial line artifact in the ABP signal) for the arterial $\mathrm{pCO}_{2}$ measurements, and the values of the reported $\mathrm{pCO}_{2}$ measurements (available in 26/29 patients).

\section{Time trends and slow wave component}

In order to isolate the slow wave (sw) component $(0.005-0.05$ $\mathrm{Hz}$ ) of the raw waveform signals, they were first decimated to sampling frequency $1 \mathrm{~Hz}$ and subsequently processed with an FFT band-pass filter. Four new signals were obtained as trends in the time domain: ABP_sw, ICP_sw, FVl_sw, and FVr_sw. In addition, power spectral density (PSD) [19] analysis was performed (periodogram method using the Hanning window) and results reported for the specified frequency range (Fig. 1). The PSD analysis allows estimation of the distribution of energy of the signal (power is energy expenditure over time) over frequency. In the context of slow waves, it simply provides a measure of variability of the analyzed signal over the specified frequency range and is calculated as an integral of the PSD function over the frequency range. For intuitive simplicity, if a square root was to be applied to the slow wave power estimations, this could be interpreted as an "equivalent amplitude" of a pure sinusoidal wave that would carry the same amount of energy. So in a sense, this parameter represents the amplitude of the slow waves.

Descriptive measures of the studied variables were extracted for each valid period per each patient. The periods were considered valid if they included at least $10 \mathrm{~min}$ of continuous data without gaps after the artifact removal and the filter application (with edge effects removed). The following metrics were calculated in the time domain: mean value, standard deviation, variance, coefficient of variation. To evaluate the complexity of the waveform, entropy was also calculated in the time domain (sample entropy, SaEn with length " $m$ " $=2$ ) [29]. Entropy is the rate of information production, a measurement of the system randomness or unpredictability. We investigated Entropy as a measure of the complexity of the time series.

In the frequency domain, limited to the studied range of frequencies, the following metrics were extracted: total power and frequency centroid. Given that the power of the slow waves is distributed in a range of frequencies rather than in one main frequency, we studied the frequency centroid as a measure of average frequency or a measure of the shape of the frequency distribution, and described its changes during hypocapnia.

In addition, $\Delta$ Power was calculated as Power baseline $_{-}$ Power hypocapnia, $_{\text {Centroid }}=$ Centroid $_{\text {baseline }}$ Centroid $_{\text {hypocapnia }}$, and $\Delta$ Entropy $=$ Entropy $_{\text {baseline }}-$ Entropy hypocapnia.

\section{Derived indexes}

Coherence between FV and ICP was calculated as the maximum coherence in the frequency range $0.005-0.05 \mathrm{~Hz}$ between the two signals in time on the $30 \mathrm{~Hz}$ data.

We calculated cerebrospinal compliance $(\mathrm{Ci})$ and the compensatory reserve index (RAP) to describe their influence in the modulation of the transmission of the vasogenic waves in the ICP waveform.

$\mathrm{Ci}$ was calculated as:

$\mathrm{Ci}=\frac{\mathrm{aCaBV}}{\mathrm{aICP}} \quad\left[\frac{\mathrm{cm}^{3}}{\mathrm{mmHg}}\right]$

with aCaBV being the Fourier amplitude of the fundamental harmonic of the pulse of the cerebral arterial blood volume $(\mathrm{CaBV}) . \mathrm{CaBV}$ was derived from FV signal:

$$
\mathrm{CaBV}(t)=\int(\mathrm{FV}(t)-\operatorname{mean}(\mathrm{FV})) d t\left[\mathrm{~cm}^{3}\right]
$$

where mean (FV) was calculated using a moving average filter (finite response filter) applied to $\mathrm{FV}[5] . \Delta \mathrm{Ci}=\mathrm{Ci}_{\text {baseline }}$ $-\mathrm{Ci}_{\text {hypocapnia. }}$

RAP was calculated as the moving correlation coefficient between slow changes in ICP pulse amplitude ( $\mathrm{aICP}=$ fundamental harmonic of the Fourier transformation of the pulse of ICP) and mean ICP (10 s average data) over a period of $5 \mathrm{~min}$, updating every minute [8]. A Fisher transformation was separately applied to RAP (RAP_FT) for the purpose of the further statistical analysis.

The mean values of coherence, $\mathrm{Ci}$ and RAP, were extracted for period 1 (baseline) and for period 2 (hypocapnia).

\section{Statistical analysis}

$\mathrm{R}$ statistical language was used to perform the statistical analysis [R: A language and environment for statistical computing. R Foundation for Statistical Computing, Vienna, Austria. URL http://www.R-project.org/. version 3.3.3]. The variables were summarized as mean values \pm SD during the baseline and the hypocapnia period. The effects of hypocapnia in the exported parameters were studied using univariate tests (paired $t$ test) comparing baseline vs hypocapnia. The correlations were performed with the Pearson method. For all tests, alpha was set at 0.05 for significance. Univariate 

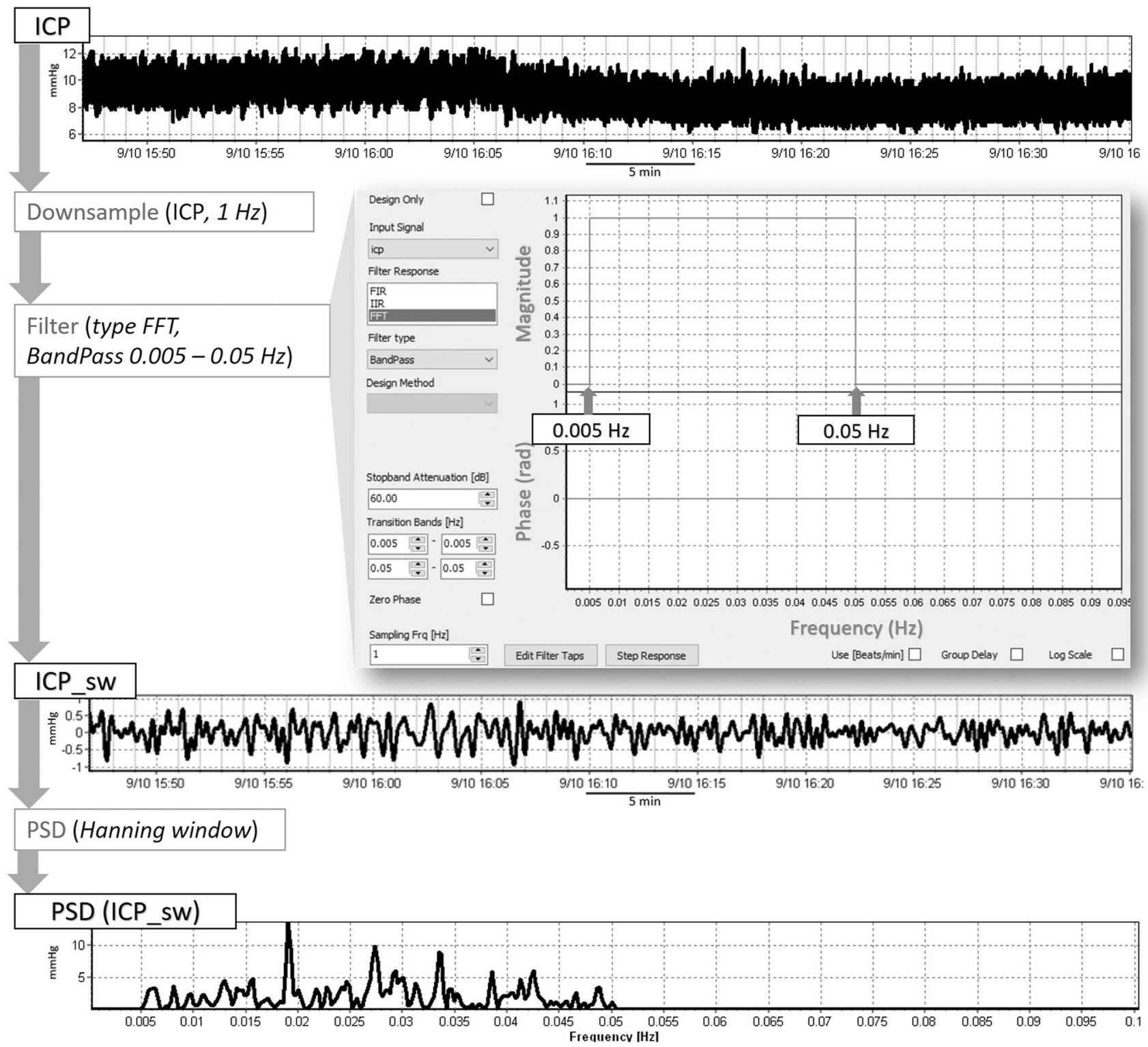

Fig. 1 Signal processing methodology applied to obtain the slow wave component of the recorded signals. The whole pipeline is shown here for ICP. The raw waveform signals (time trend at the top) were first decimated to sampling frequency $1 \mathrm{~Hz}$ and subsequently processed

and multivariate linear and non-linear models were explored, assessing superiority with Akaike Information Criterion (AIC).

\section{Results}

Twenty-nine adult (median age 39 years, 5 females) severe TBI patients (mean GCS $6 \pm 3$ ) were included. Figure 2 shows a typical example of the time trends of the recorded signals and Fig. 3 shows an example of the trend of the calculated slow waves component and the PSD chart during the baseline and the hypocapnia period, where mean $\mathrm{pCO}_{2}$ dropped from with a FFT band-pass filter designed as shown in the picture, in order to isolate the slow wave component $(0.005-0.05 \mathrm{~Hz})$ of the four waveforms. Power spectral density (PSD) analysis was performed (periodogram method with the settings as shown in the picture)

$5.10 \pm 0.36 \mathrm{kPa}$ to $4.39 \pm 0.35 \mathrm{kPa}, p<0.001$. Statistical comparisons of baseline versus hypocapnic period values are given in Table 1. Correlations between changes in ICP slow wave pattern and other physiological variables are presented in Table 2.

\section{Influence of hypocapnia on slow waves}

According to the visual assessment of the time trends (Fig. 3), the slow wave component of ICP decreased in magnitude in $23 / 29$ patients and did not change in $2 / 29$.

The results of the statistical comparison baseline vs hypocapnia (Table 1) showed that this mainly affects ICP 


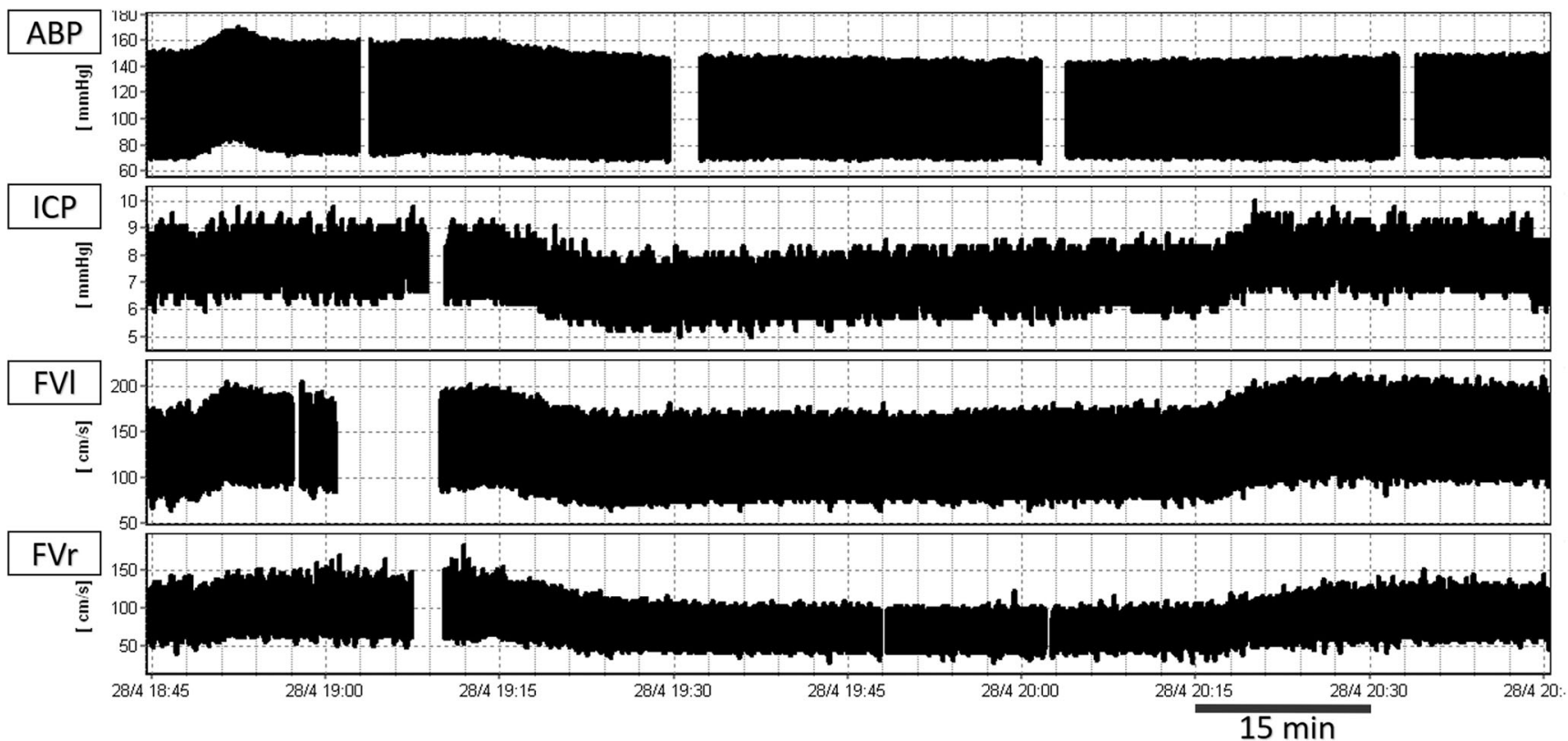

Fig. 2 Example of the time trends of the recorded signals. Sampling frequency $=30 \mathrm{~Hz}$. The gaps in the charts are due to the manual removal of artifacts such as arterial line flush, ICP transients, or FV disturbed signals. ABP, arterial blood pressure; ICP, intracranial pressure; FVl, flow velocity left; FVr, flow velocity right

variable with a significant change in variance of its mean value in the time domain. FVr-related parameters have a deeper decrease in hypocapnia if compared with the left side, except for the centroid and entropy of slow waves. The

and therefore entropy of slow waves. ICP was the only

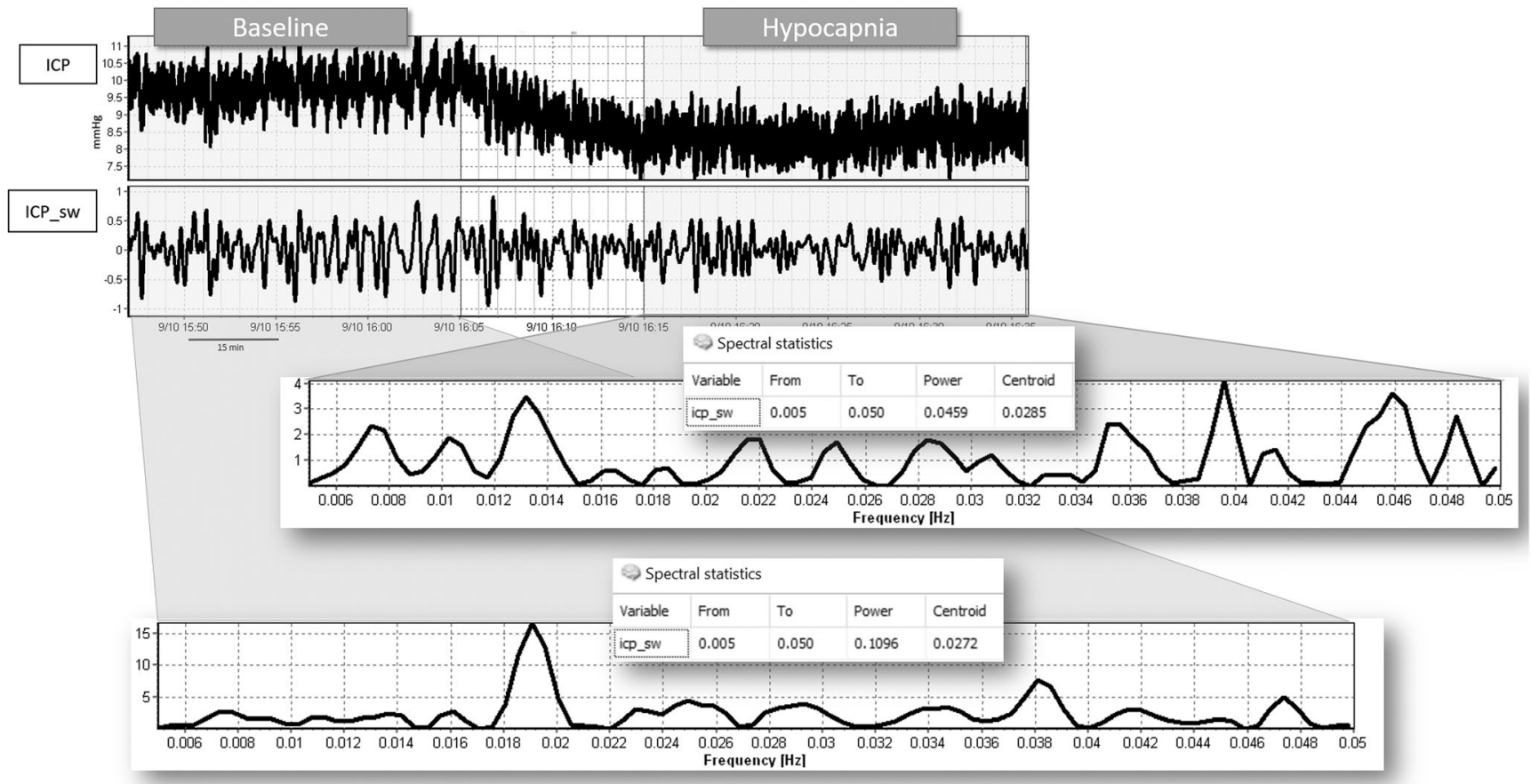

Fig. 3 Time trend of the calculated ICP slow wave component and the PSD chart during the baseline and the hypocapnia period. ICP time series is presented as resampled to $1 \mathrm{~Hz}$. ICP_sw shows the trend of the slow wave component of intracranial pressure as obtained by applying the FFT band-pass filter. Power spectral density analysis is shown (periodogram method using Hanning window) for the specific frequency range, for the two selected periods (baseline and hypocapnia). The correspondent power and centroid are shown in the spectral statistics table. ICP, intracranial pressure; ICP sw, intracranial pressure slow waves time series; PSD, power spectral density 
Table 1 Mean values and SD of the studied parameters at the baseline and during hypocapnia. When the changes between baseline and hypocapnia are significant, the direction of the change is highlighted. $p$ values of univariate analysis are quoted uncorrected for multiple comparison

\begin{tabular}{|c|c|c|c|c|c|c|c|c|}
\hline \multirow[t]{2}{*}{ Variable } & & & \multicolumn{2}{|c|}{ Baseline } & \multicolumn{2}{|c|}{ Hypocapnia } & \multirow[t]{2}{*}{ Change } & \multirow[t]{2}{*}{$p$ value } \\
\hline & & & Mean & SD & Mean & SD & & \\
\hline \multirow[t]{8}{*}{ Frequency domain } & \multirow[t]{4}{*}{ Power of sw $\left(\mathrm{mmHg}^{2}\right)$} & ICP & 1.04 & 1.82 & 0.27 & 0.49 & 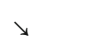 & $0.006^{*}$ \\
\hline & & $\mathrm{ABP}$ & 2.95 & 4.14 & 1.90 & 1.40 & & 0.177 \\
\hline & & FV1 & 16.02 & 20.48 & 10.90 & 13.66 & $\searrow$ & $0.049 *$ \\
\hline & & $\mathrm{FVr}$ & 16.88 & 21.69 & 9.48 & 11.67 & $\searrow$ & $0.005^{*}$ \\
\hline & \multirow[t]{4}{*}{ Centroid of sw (Hz) } & $\mathrm{ICP}$ & 0.020 & 0.006 & 0.023 & 0.005 & $\pi$ & $<0.001 *$ \\
\hline & & $\mathrm{ABP}$ & 0.016 & 0.005 & 0.020 & 0.006 & $\pi$ & $<0.001 *$ \\
\hline & & $\mathrm{FVl}$ & 0.023 & 0.006 & 0.027 & 0.004 & $\pi$ & $<0.001 *$ \\
\hline & & $\mathrm{FVr}$ & 0.022 & 0.006 & 0.026 & 0.005 & $\pi$ & $<0.001 *$ \\
\hline \multirow[t]{4}{*}{ Time domain, sw } & \multirow[t]{4}{*}{ Entropy of sw } & $\mathrm{ICP}$ & 0.50 & 0.11 & 0.56 & 0.06 & $\pi$ & $<0.001 *$ \\
\hline & & $\mathrm{ABP}$ & 0.44 & 0.11 & 0.51 & 0.10 & $\pi$ & $<0.001 *$ \\
\hline & & FV1 & 0.55 & 0.07 & 0.58 & 0.05 & $\pi$ & $<0.001 *$ \\
\hline & & $\mathrm{FVr}$ & 0.54 & 0.09 & 0.58 & 0.07 & $\pi$ & $<0.001 *$ \\
\hline \multirow[t]{12}{*}{ Time domain, time series } & \multirow[t]{4}{*}{ Time series (mmHg) } & $\mathrm{ICP}$ & 16.65 & 6.70 & 13.03 & 6.35 & 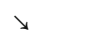 & $<0.001 *$ \\
\hline & & $\mathrm{ABP}$ & 96.45 & 8.98 & 98.83 & 11.65 & & 0.100 \\
\hline & & $\mathrm{FVl}$ & 77.67 & 35.65 & 65.57 & 29.72 & 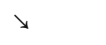 & $<0.001 *$ \\
\hline & & $\mathrm{FVr}$ & 78.14 & 26.82 & 61.65 & 17.50 & $\searrow$ & $<0.001 *$ \\
\hline & \multirow[t]{4}{*}{ Coefficient of variation } & ICP & 0.09 & 0.05 & 0.10 & 0.08 & & 0.206 \\
\hline & & $\mathrm{ABP}$ & 0.04 & 0.02 & 0.04 & 0.02 & & 0.936 \\
\hline & & FVl & 0.07 & 0.03 & 0.08 & 0.04 & & 0.071 \\
\hline & & $\mathrm{FVr}$ & 0.07 & 0.03 & 0.08 & 0.04 & & 0.249 \\
\hline & \multirow[t]{4}{*}{ Variance } & $\mathrm{ICP}$ & 2.23 & 2.16 & 1.39 & 1.39 & $\searrow$ & $0.006^{*}$ \\
\hline & & ABP & 14.94 & 13.29 & 17.10 & 32.21 & & 0.700 \\
\hline & & FVl & 37.10 & 36.29 & 39.37 & 71.75 & & 0.825 \\
\hline & & $\mathrm{FVr}$ & 40.89 & 41.65 & 37.83 & 72.34 & & 0.869 \\
\hline \multirow[t]{5}{*}{ Time domain, derivate indexes } & \multicolumn{2}{|l|}{ Coherence ICP-FVl } & 0.78 & 0.19 & 0.78 & 0.19 & & 0.951 \\
\hline & \multicolumn{2}{|l|}{ Coherence ICP-FVr } & 0.76 & 0.21 & 0.76 & 0.19 & & 0.401 \\
\hline & \multicolumn{2}{|l|}{$\mathrm{Ci} 1\left(\mathrm{~cm}^{3} / \mathrm{mmHg}\right)$} & 2.15 & 1.41 & 3.08 & 2.38 & $\pi$ & $0.006^{*}$ \\
\hline & \multicolumn{2}{|l|}{$\mathrm{Ci}$ r $\left(\mathrm{cm}^{3} / \mathrm{mmHg}\right)$} & 2.36 & 1.63 & 3.07 & 2.69 & $\pi$ & $0.008^{*}$ \\
\hline & \multicolumn{2}{|l|}{ RAP } & 0.51 & 0.34 & 0.41 & 0.34 & $\searrow$ & $0.026^{*}$ \\
\hline
\end{tabular}

$s w$ slow waves, $I C P$ intracranial pressure, $A B P$ arterial blood pressure, $F V l$ flow velocity left, $F V r$ flow velocity right, $C i \_l$ compliance of cerebrospinal space left, $C i \_r$ compliance of cerebrospinal fluid right, $R A P$ compensatory reserve index

* denotes statistically significant finding

coefficient of variation did not change significantly, yet there was a tendency to increase during hypocapnia.

\section{Description of the correlation ICP-FV slow waves}

The coherence between FV and ICP in the slow wave range was high $(>0.7)$ both at the baseline and during hypocapnia, with the left side coherence seemingly decreasing and the right side seemingly increasing during hypocapnia, but changes were not significant (Table 1). No statistical significance was found when ICP and FV slow waves-related metrics were correlated, except for changes in entropy at the left side and for absolute value of centroid on the right side (Table 2).

\section{Description of the correlation with compliance of brain CSF (Ci) space and compensatory reserve (RAP)}

$\mathrm{Ci}$ increased significantly in the mean value from baseline to hypocapnia, while RAP decreased (Table 1). No statistical significance was found between changes in ICP slow waves and $\mathrm{Ci}$ (Table 2). RAP_FT was used for the following correlations (Table 2). The delta power of ICP slow waves was correlated to the delta RAP and so did the mean value of RAP at the baseline with even stronger correlation (Fig. 4). Delta RAP at the baseline was not correlated with the delta power of FV slow waves on the left side, but it was on the right side. 
Table 2 Correlations between changes in ICP slow waves pattern and other physiological variables. The analysis is performed both for the changes between baseline and hypocapnia, and for the hypocapnic absolute values. The $p$ values were not adjusted for multiple comparisons

\begin{tabular}{|c|c|c|c|}
\hline & Variables & $r$ & $p$ \\
\hline \multirow{6}{*}{$\begin{array}{l}\text { ICP-FV } \\
\text { (baseline-hypocapnia) }\end{array}$} & $\Delta P_{\mathrm{ICP}} ; \Delta P_{\mathrm{FVl}}$ & 0.15 & 0.44 \\
\hline & $\Delta P_{\mathrm{ICP}} ; \Delta P_{\mathrm{FVr}}$ & 0.33 & 0.08 \\
\hline & $\Delta C_{\mathrm{ICP}} ; \Delta C_{\mathrm{FVl}}$ & 0.27 & 0.17 \\
\hline & $\Delta C_{\mathrm{ICP}} ; \Delta C_{\mathrm{FVr}}$ & 0.30 & 0.12 \\
\hline & $\Delta E_{\mathrm{ICP}} ; \Delta E_{\mathrm{FVl}}$ & 0.38 & 0.04 \\
\hline & $\Delta E_{\mathrm{ICP}} ; \Delta E_{\mathrm{FVr}}$ & 0.32 & 0.09 \\
\hline \multirow{6}{*}{$\begin{array}{l}\text { ICP-FV } \\
\text { (during hypocapnia) }\end{array}$} & $P_{\mathrm{ICP}} ; P_{\mathrm{FVl}}$ & 0.05 & 0.80 \\
\hline & $P_{\mathrm{ICP}} ; P_{\mathrm{FVr}}$ & 0.06 & 0.74 \\
\hline & $C_{\mathrm{ICP}} ; C_{\mathrm{FVl}}$ & 0.21 & 0.27 \\
\hline & $C_{\mathrm{ICP}} ; C_{\mathrm{FVr}}$ & 0.40 & 0.03 \\
\hline & $E_{\mathrm{ICP}} ; E_{\mathrm{FVl}}$ & 0.14 & 0.46 \\
\hline & $E_{\mathrm{ICP}} ; E_{\mathrm{FVr}}$ & 0.31 & 0.10 \\
\hline \multirow{2}{*}{$\begin{array}{l}\text { ICP-Ci } \\
\text { (during hypocapnia) }\end{array}$} & $P_{\mathrm{ICP}} ; \mathrm{Ci}_{1}$ & -0.11 & 0.58 \\
\hline & $P_{\mathrm{ICP}} ; \mathrm{Ci}_{\mathrm{r}}$ & -0.14 & 0.47 \\
\hline \multirow{2}{*}{$\begin{array}{l}\text { ICP-Ci } \\
\text { (baseline-hypocapnia) }\end{array}$} & $\Delta P_{\mathrm{ICP}} ; \Delta \mathrm{Ci}_{1}$ & 0.15 & 0.42 \\
\hline & $\Delta P_{\mathrm{ICP}} ; \Delta \mathrm{Ci}_{\mathrm{r}}$ & 0.14 & 0.48 \\
\hline \multirow[t]{2}{*}{ ICP-RAP } & $\Delta P_{\mathrm{ICP}} ; \Delta \mathrm{RAP}$ & 0.58 & 0.001 \\
\hline & $\Delta P_{\mathrm{ICP}} ; \mathrm{RAP}_{\mathrm{b}}$ & 0.71 & $<0.001$ \\
\hline \multirow[t]{2}{*}{ FV-RAP } & $\Delta P_{\mathrm{FVl}} ; \Delta \mathrm{RAP}$ & 0.26 & 0.18 \\
\hline & $\Delta P_{\mathrm{FVr}} ; \Delta \mathrm{RAP}$ & 0.50 & 0.007 \\
\hline
\end{tabular}

$\Delta$ absolute delta value calculated as baseline-hypocapnia, $P$ power of the slow waves, $E$ entropy of the slow waves, $C$ centroid of the slow waves, $I C P$ intracranial pressure, $F V l$ flow velocity left, $F V r$ flow velocity right, Cil cerebrospinal compliance on the left side, $C i r$ cerebrospinal compliance on the right side, $R A P$ compensatory reserve index, $R A P b$ compensatory reserve at the baseline

A multiple linear regression model incorporating all the physiological variables (RAP at the baseline $\left(\mathrm{RAP}_{\mathrm{b}}\right)$, delta $\mathrm{Ci}$ and delta power of FV slow waves) was investigated in order to identify factors that could better predict the decrease in the power of ICP slow waves. The results suggested RAP at the baseline $(p<0.001)$ as the strongest predictor for the decrease in the power of ICP slow waves $\left(\Delta P_{\mathrm{ICP}}\right)$. Other, reduced, models were compared with the full one, and the superiority was assessed with AIC. If only $\mathrm{RAP}_{\mathrm{b}}$ was considered in the model (non-linear), $\Delta P_{\mathrm{ICP}}$ could be described as

$\Delta P_{\mathrm{ICP}}=-1.3+2.1^{\mathrm{RAPb}}$

This showed the best fit according to the AIC test and expressed $67 \%$ of the total variation of the drop in power of ICP slow waves (Fig. 4).

\section{Discussion}

In this study, we intended to scrutinize the behavior of the slow waves of ICP during short hypocapnia tests in TBI patients and to relate it to the other relevant physiological variables.

Given the physiological information carried by the ICP slow waves, several different methods have been proposed for their qualitative and quantitative analysis. Eklund et al. [11] described two computerized methods, one in the time domain (waveform analysis) and one in the frequency domain (estimation of B waves power in 10 min blocks of ICP monitoring). Hara et al. [13] used an automated offline detection of slow waves based on the power spectrum of ICP oscillations by fast Fourier transform (FFT), while Walter et al. [38] proposed an online version based on an ARMA modeling derived spectral estimation. Kasprowicz et al. [18] focused on the analysis of the morphology changes of individual ICP pulses during the slow waves. Spiegelberg et al. [34] applied a pattern recognition in the time domain. However, there is not any apparent advantage of using one method over another and the

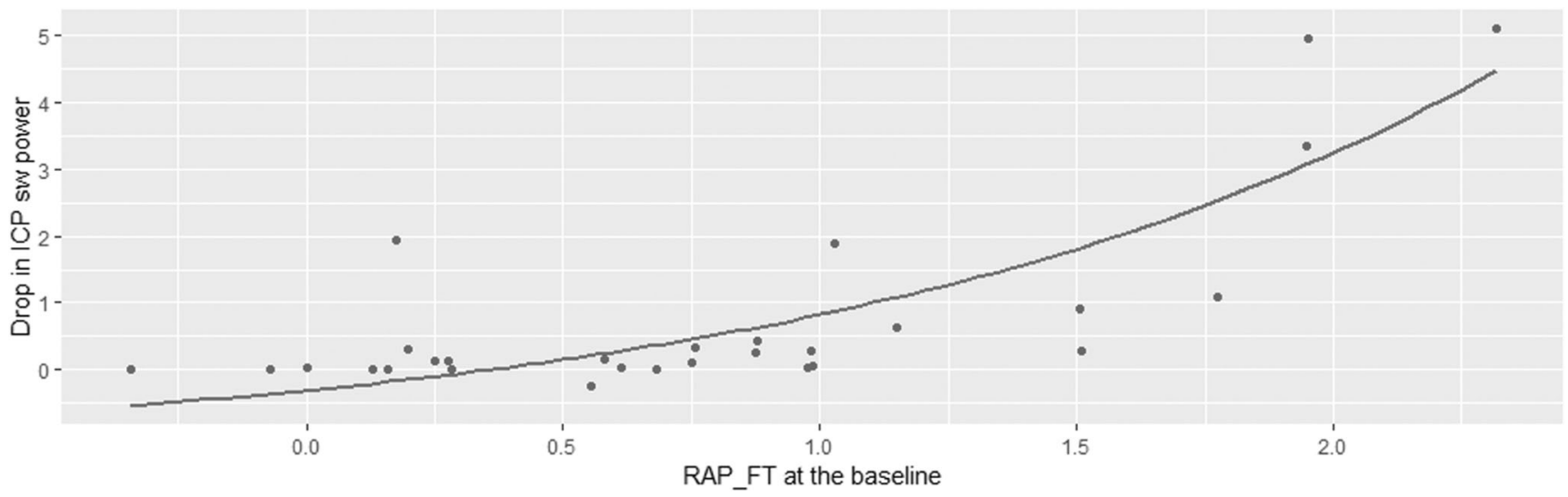

Fig. 4 Relationship between RAP at the baseline (using the RAP fisher transformed) and the drop in power in ICP slow waves during hypocapnia $\left(\Delta\right.$ Power $=$ Power $_{\text {baseline }}-$ Power $\left._{\text {hypocapnia }}\right)$. This can be described as
$\Delta P_{\mathrm{ICP}}=-1.3+2.1^{\mathrm{RAPb}} ;$ adjusted $R$ squared $=0.67 . \mathrm{ICP}$, intracranial pressure; sw, slow waves; RAP FT, compensatory index RAP after Fisher transformation 
description of the changes in slow waves in different conditions depends on the methods utilized. We therefore firstly defined a methodology that allowed us to approach the slow waves both in the time and frequency domains and to describe them in terms of total power, frequencies patterns, and time domain variability in our dataset. We defined the slow waves in terms of their frequency, range of 0.005 to $0.05 \mathrm{~Hz}$, not specifying a minimum value for their amplitude.

As expected from the visual inspection, the spectral power of the slow waves decreased significantly in ICP and in FV during hypocapnia when compared with the baseline period. However, the range of frequencies where the power was concentrated was widened with a shift of the center of gravity toward the higher frequencies (an increase of the centroid of the slow waves). Overall, if the activity of the slow waves is defined only by the total power, then we can state that hypocapnia decreases the slow waves activity. But, if we consider the frequency distribution characteristics as part of the slow waves activity signature, then this is perhaps not entirely true anymore, with the centroid change indicating increase in the higher frequency content of the waves (more "complex" waveform morphology), counterbalancing, in a way, the power decrease.

The supposition of a more complex waveform pattern during hypocapnia seems to be confirmed by the observed increase in the sample entropy calculated in the time domain of the slow waves. The meaning of this needs to be clarified. In biological systems, higher entropy of the relevant physiological measurement time series usually indicates a healthier system, while a breakdown in homeostasis usually leads to its decrease [12]-[28]-[33]. In our dataset, suddenly induced mild-moderate hypocapnia increased the entropy related to the slow waves, suggesting a potential improvement in the regulatory capacity of the whole cerebrovascular system.

The power of ABP slow waves did not seem to be affected by hypocapnia since the decrease did not reach a statistical significance. This suggests that the observed changes in ICP and FV slow waves pattern were either due to changes in the transmission of the $\mathrm{ABP}$ waves to $\mathrm{CBV}$, or that they were caused by an independent, intracranial, cerebrovascular tone modulating mechanism affected by hypocapnia. However, the fact that the frequency composition/waveform morphology showed the same pattern of change as the other two modalities (as indicated by the centroid and entropy metrics) seems to favor the former interpretation.

Induced hypocapnia causes, with intact cerebrovascular reactivity, a cerebral vasoconstriction, which ultimately leads to a decrease in CBV and thus ICP. As cerebrovascular resistance increases, a decrease in FV will follow if significant changes in ABP do not occur (Fig. 5). The vasogenic waves generated by the dynamic changes in $\mathrm{CBV}$ at the two vascular diameter levels (relatively dilated baseline and relatively constricted hypocapnia) will have different amplitude depending on the vascular tone (a fall in the vascular tone causes an increase in the amplitude of the vascular waves) [1]. A parameter called vascular "wall tension" (WT) is considered an indicator of the arterial smooth muscles tones. This parameter has been investigated in the same data set by Smielewski et al. in a previous study: WT was shown to increase during hypocapnia, leading to a stiffer arterial bed and thus attenuated transmission of arterial pressure waves. In the same study, the cerebrovascular reactivity was shown to be intact for both levels of $\mathrm{CO} 2$, with no significant difference between the two [32].

We further considered the transmission of the generated vasogenic slow waves into FV [25] and in ICP waveforms [1]. FV and ICP slow waves activity shows a similar pattern change after the suddenly induced hypocapnia if compared with the preceding baseline period (decreased power and increased centroid frequency), which might be explained by the common origin of the vasogenic waves. However, we did not find a significant correlation between the slow waves related metrics in ICP and FV. The power modification in the FV slow waves might be explained by the fact that ABP slow waves power also showed a tendency to decrease during hypocapnia, even if that did not quite reach statistical significance. Clearly, a different mechanism from the variation in the CBV fluctuations must be responsible for the modification in the ICP slow waves pattern during hypocapnia.

What changes here is probably the "transmission factor," related to cerebrospinal compliance and compensatory reserve (Fig. 5). The amplitude of the ICP slow waves generally increases in low compensatory reserve [39] and Lundeberg postulated their rise in low compliance [24]. Given that a decrease in CBV causes an improvement in the compliance (which we also observed), we investigated the relationship between cerebral compliance and the hypocapnia induced variation in the ICP slow waves pattern.

We did not find a correlation between the variation in total power of ICP slow waves and the $\mathrm{Ci}$, which suggests that the CSF and venous compartment are not the only ones playing a role in the decrease in the power of ICP slow waves. Interestingly, the drop in power of the ICP slow waves was correlated to the increase in compensatory reserve from baseline to hypocapnia and more strongly to the baseline compensatory reserve. RAP is a more comprehensive index then $\mathrm{Ci}$, because it takes into account the multiple cerebral compartments. The worse the compensatory reserve at the baseline, meaning the tighter the brain before the hypocapnic challenge, the higher the difference in the power of ICP slow waves induced by hypocapnia. We suggest here a nonlinear model describing this relationship (Fig. 4), where variation in the power of ICP slow waves increases exponentially with RAP at the baseline. Similarly, Steiner et al. [35] showed in the same dataset that RAP at the baseline was the strongest predictor of the reduction of ICP mean value during hypocapnia. 
Fig. 5 Mechanisms involved in the generation and transmission of the slow waves in the intracranial vault. $\mathrm{ABP}$, arterial blood pressure; ICP, intracranial pressure; $\mathrm{CPP}$, cerebral perfusion pressure; $\mathrm{CA}$, cerebral autoregulation; CVR, cerebrovascular resistance, $\mathrm{CBV}$, cerebral blood volume; Met, metabolic factors; $\mathrm{PaCO} 2$, arterial carbon dioxide pressure; RAP, compensatory reserve index; $\mathrm{CBF}$, cerebral blood flow

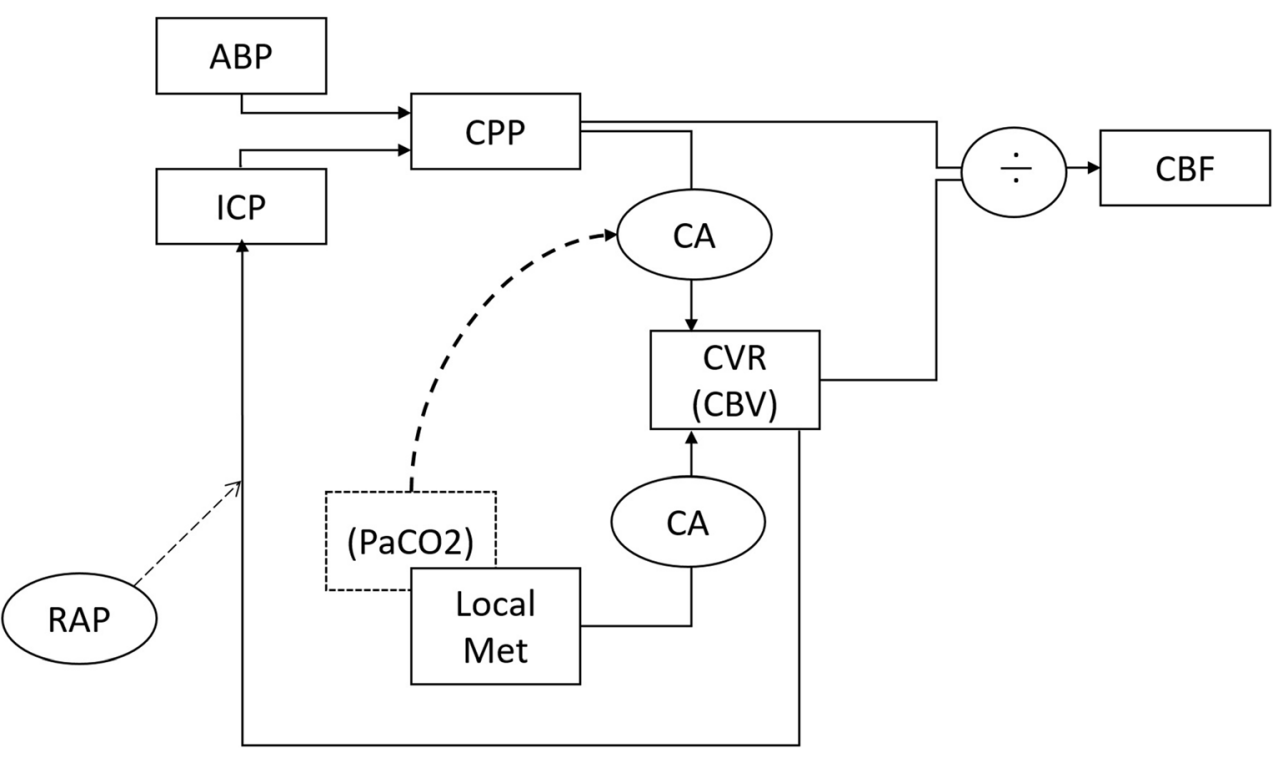

In TBI patients, therefore, induced mild to moderate hypocapnia provokes on the one hand a decrease in ICP slow waves power (the magnitude of which depends on the compensatory reserve during the previous normocapnia period) and on the other hand an increase of the ICP slow wave centroid (increased higher frequencies contribution) and improvement of their entropy. Quantifying these relationships may be clinically relevant when the metrics derived from the slow waves analysis, such as cerebral autoregulation (via assessment of the pressure reactivity index PRx) [36], brain stem activity [22],[15], and cerebral compliance [24, 27], are used in daily monitoring and integrated with the other clinical diagnostic "tools." The change in power may need to be correlated to the state of the compensatory reserve and perhaps should be taken into account when interpreting the derived metrics, given the correlation with the power of slow waves and clinical outcome. The variability induced in the morphological content on the other hand could provide further insights into the cerebrovascular system, confirming the nonharmful effect of short term hypocapnic challenges used for treating intracranial hypertension.

\section{Limitations}

In this retrospective study, only 29 patients were investigated and the patient heterogeneity as well as the injury pattern heterogeneity was not taken into account. Further studies with a larger cohort of patients will be needed to validate these preliminary findings.

Although the ventilator settings were kept constant during the hypocapnia challenge and during the period directly preceding it, we cannot exclude small, but possibly influential, variations in $\mathrm{PaCO}_{2}$. Having $\mathrm{EtCO}_{2}$ measurements available with our data set would go some way toward further reassurance of stable $\mathrm{PaCO}_{2}$ levels.

The hypocapnic periods were selected in the very first hypocapnic period after the stabilization; therefore, our findings might not reflect what happens in late hypocapnia. Further investigations are required to describe the behavior of intracranial slow waves in late hypocapnia.

In the same way, deep hypocapnia may show different results vs mild hypocapnia. Therefore, changes in slow waves during deep hypocapnia should be studied separately.

From the methodological point of view, the artifacts and in particular the "transient" patterns were defined heuristically by the investigators. However, the choice was kept consistent. For future studies, an agreement on the artifacts and transients definition should be achieved.

Moreover, even if the description of the pattern of the slow waves during the immediate post-hypocapnia period would have been desirable and informative, a clear posthypocapnia period could not be identified in a reliable way and a comparison hypocapnia vs post-hypocapnia state was not possible.

\section{Conclusions}

We found that in severe TBI patients, a sudden mild to moderate hypocapnia induces a decrease in ICP and FV slow wave power. It also increases their higher frequency content and their morphological complexity (entropy). The difference in power of the ICP slow waves between the baseline and the hypocapnia period depends on the baseline compensatory reserve, as expressed by RAP index.

Acknowledgments We thank Andras Czigler for his technical suggestions and his help with editing the manuscript. 


\section{Compliance with ethical standards}

Conflict of interests PS and MCz receive part of the licensing fees for multimodal brain monitoring software ICM+, licensed by Cambridge Enterprise Ltd., University of Cambridge, UK. There are no other conflicts of interest to declare.

Ethical approval As previously mentioned (section Materials and Methods), all procedures involving human participants were in accordance with the ethical standards of the institutional and national research committee (UK Health Departments (2011) Governance arrangements for research ethics committees) and with the 1964 Helsinki declaration and its later amendments or comparable ethical standards. For this type of study formal consent is not required.

Open Access This article is distributed under the terms of the Creative Commons Attribution 4.0 International License (http:// creativecommons.org/licenses/by/4.0/), which permits unrestricted use, distribution, and reproduction in any medium, provided you give appropriate credit to the original author(s) and the source, provide a link to the Creative Commons license, and indicate if changes were made.

\section{References}

1. Auer LM, Sayama I (1983) Intracranial pressure oscillations (Bwaves) caused by oscillations in cerebrovascular volume. Acta Neurochir 68(1-2):93-100

2. Balestreri M, Czosnyka M, Steiner LA, Schmidt E, Smielewski P, Matta B, Pickard JD (2004) Intracranial hypertension: what additional information can be derived from ICP waveform after head injury? Acta Neurochir 146(2):131-141

3. Brady KM, Easley RB, Kibler K et al (2012) Positive endexpiratory pressure oscillation facilitates brain vascular reactivity monitoring. J Appl Physiol 113(9):1362-1368

4. Brian JE (1998) Carbon dioxide and the cerebral circulation. Anesthesiology 88(5):1365-1386

5. Carrera E, Steiner LA, Castellani G, Smielewski P, Zweifel C, Haubrich C, Pickard JD, Menon DK, Czosnyka M (2011) Changes in cerebral compartmental compliances during mild hypocapnia in patients with traumatic brain injury. J Neurotrauma 28(6):889-896

6. Coles JP, Fryer TD, Coleman MR et al (2007) Hyperventilation following head injury: effect on ischemic burden and cerebral oxidative metabolism. Crit Care Med 35(2):568-578

7. Czosnyka M, Pickard JD (2004) Monitoring and interpretation of intracranial pressure. J Neurol Neurosurg Psychiatry 75(6):813821

8. Czosnyka M, Price DJ, Williamson M (1994) Monitoring of cerebrospinal dynamics using continuous analysis of intracranial pressure and cerebral perfusion pressure in head injury. Acta Neurochir 126(2-4):113-119

9. Czosnyka M, Smielewski P, Kirkpatrick P, Laing RJ, Menon D, Pickard JD (1997) Continuous assessment of the cerebral vasomotor reactivity in head injury. Neurosurgery 41(1):11-17 discussion $17-9$

10. Einhäupl KM, Garner C, Dirnagl U, Schmieder G, Schmiedek P, Kufner G, Rieder J (1986) Oscillations of ICP related to cardiovascular parameters BT - intracranial pressure VI. In: Miller JD, Teasdale GM, Rowan JO, Galbraith SL, Mendelow AD (eds). Springer Berlin Heidelberg, Berlin, Heidelberg, pp 290-297
11. Eklund A, Agren-Wilsson A, Andersson N, Bergenheim AT, Koskinen LO, Malm J (2001) Two computerized methods used to analyze intracranial pressure B waves: comparison with traditional visual interpretation. J Neurosurg 94(3):392-396

12. Goldstein B, Fiser DH, Kelly MM, Mickelsen D, Ruttimann U, Pollack MM (1998) Decomplexification in critical illness and injury: relationship between heart rate variability, severity of illness, and outcome. Crit Care Med 26(2):352-357

13. Hara K, Nakatani S, Ozaki K, Ikeda T, Mogami H (1990) Detection of the B waves in the oscillation of intracranial pressure by fast Fourier transform. Med Inform 15(2):125-131. https://doi.org/10. 3109/14639239008997664

14. Harper AM (1966) Autoregulation of cerebral blood flow: influence of the arterial blood pressure on the blood flow through the cerebral cortex. J Neurol Neurosurg Psychiatry 29(5):398-403

15. Higashi S, Yamamoto S, Hashimoto M, Fujii H, Ito H, Kogure Y, Tokuda K (1989) The role of vasomotor center and adrenergic pathway in B-waves BT - intracranial pressure VII. In: Hoff JT, Betz AL (eds). Springer, Berlin Heidelberg, Berlin, Heidelberg, pp 220-224

16. Hundley WG, Renaldo GJ, Levasseur JE, Kontos HA (1988) Vasomotion in cerebral microcirculation of awake rabbits. Am J Physiol 254(1 Pt 2):H67-H71

17. Jones SC, Williams JL, Shea M, Easley KA, Wei D (1995) Cortical cerebral blood flow cycling: anesthesia and arterial blood pressure. Am J Physiol 268(2 Pt 2):H569-H575

18. Kasprowicz M, Asgari S, Bergsneider M, Czosnyka M, Hamilton R, Hu X (2010) Pattern recognition of overnight intracranial pressure slow waves using morphological features of intracranial pressure pulse. J Neurosci Methods 190(2):310-318

19. Kay SM (1988) Modern spectral estimation: theory and application. Prentice Hall

20. Kuo TB-J, Chern C-M, Sheng W-Y, Wong W-J, Hu H-H (1998) Frequency domain analysis of cerebral blood flow velocity and its correlation with arterial blood pressure. J Cereb Blood Flow Metab 18(3):311-318

21. Lalou DA, Czosnyka M, Donnelly J, Lavinio A, Pickard JD, Garnett M, Czosnyka Z (2016) Influence of general anaesthesia on slow waves of intracranial pressure. Neurol Res 38(7):587-592

22. Lang EW, Diehl RR, Timmermann L, Baron R, Deuschl G, Mehdorn HM, Zunker P (1999) Spontaneous oscillations of arterial blood pressure, cerebral and peripheral blood flow in healthy and comatose subjects. Neurol Res 21(7):665-669

23. Lu C-W, Czosnyka M, Shieh J-S, Pickard JD, Smielewski P (2016) Acta Neurochir Suppl 122:33-35

24. Lundberg N (1960) Continuous recording and control of ventricular fluid pressure in neurosurgical practice. Acta Psychiatr Scand Suppl 36(149):1-193

25. Mautner-Huppert D, Haberl RL, Dirnagl U, Villringer A, Schmiedek P, Einhäupl K (1989) B-waves in healthy persons. Neurol Res 11(4):194-196

26. Muizelaar JP, Marmarou A, Ward JD, Kontos HA, Choi SC, Becker DP, Gruemer H, Young HF (1991) Adverse effects of prolonged hyperventilation in patients with severe head injury: a randomized clinical trial. J Neurosurg 75(5):731-739

27. Newell DW, Aaslid R, Stooss R, Reulen HJ (1992) The relationship of blood flow velocity fluctuations to intracranial pressure B waves. J Neurosurg 76(3):415-421

28. Norris PR, Anderson SM, Jenkins JM, Williams AE, Morris JA (2008) Heart rate multiscale entropy at three hours predicts hospital mortality in 3,154 trauma patients. Shock (Augusta, Ga) 30(1):1722

29. Richman JS, Moorman JR (2000) Physiological time-series analysis using approximate entropy and sample entropy. Am J Physiol Heart Circ Physiol 278(6):H2039-H2049 
30. Rosner MJ (1986) The vasodilatory cascade and intracranial pressure. In: Miller JD, Teasdale GM, Rowan JO, Galbraith SL, Mendelow AD (eds) Intracranial Pressure VI. Springer, Berlin Heidelberg, Berlin, Heidelberg, pp 137-141

31. Russo G, Lodi CA, Ursino M (2000) Quantitative assessment of cerebral vascular reserve by means of transcranial Doppler ultrasound and rebreathing maneuver: bedside test and mathematical modeling. Neurol Sci 21(5):292-302

32. Smielewski P, Steiner LA, Puppo C, Budohoski K, Varsos GV, Czosnyka M (2018) Effect of mild hypocapnia on critical closing pressure and other mechanoelastic parameters of the cerebrospinal system. Acta Neurochir Suppl 126:139-142

33. Sortica da Costa C, Placek MM, Czosnyka M, Cabella B, Kasprowicz M, Austin T, Smielewski P (2017) Complexity of brain signals is associated with outcome in preterm infants. J Cereb Blood Flow Metab 37(10):3368-3379

34. Spiegelberg A, Krause M, Meixensberger J, Seifert B, Kurtcuoglu V (2018) Significant association of slow vasogenic ICP waves with normal pressure hydrocephalus diagnosis. Acta Neurochir Suppl: 243-246

35. Steiner LA, Balestreri M, Johnston AJ, Coles JP, Smielewski P, Pickard JD, Menon DK, Czosnyka M (2005) Predicting the response of intracranial pressure to moderate hyperventilation. Acta Neurochir 147(5):477-483

36. Steinmer R, Rauhuf C, Hübner U, Bauer R, Fahlbusch R, Laumer R, Bondar I (1996) Slow rhythmic oscillations of blood pressure, intracranial pressure, microcirculation, and cerebral oxygenation. Dynamic interrelation and time course in humans. Stroke 27(12): 2236-2243

37. Venes JL (1979) B waves - a reflection of cardiorespiratory or cerebral nervous systems rhythm? Pediatr Neurosurg 5(3):352-360

38. Walter M, Kiefer M, Leonhardt S, Steudel WI, Isermann R (2002) Online analysis of intracranial pressure waves. Acta Neurochir Suppl 81:161-162
39. Weerakkody RA, Czosnyka M, Zweifel C, Castellani G, Smielewski P, Keong N, Haubrich C, Pickard J, Czosnyka Z (2010) Slow vasogenic fluctuations of intracranial pressure and cerebral near infrared spectroscopy-an observational study. Acta Neurochir 152(10): 1763-1769

40. Yoshihara M, Bandoh K, Marmarou A (1995) Cerebrovascular carbon dioxide reactivity assessed by intracranial pressure dynamics in severely head injured patients. J Neurosurg 82(3):386-393

\section{Comments}

The authors have analyzed the influence of hyperventilation on ICP slow waves (earlier described as B waves by Lundberg) in 29 TBI patients, by comparing baseline and hypocapnic challenge periods. They found among other findings that hypocapnia induced a decrease in ICP and flow velocity slow waves power with increased frequency content and morphological complexity. The decrease of ICP slow wave power depended on baseline compensatory reserve (RAP). The findings suggest that induced mild-moderate hyperventilation may contribute to a healthier cerebrovascular system and increased compensatory volume reserve. Better interpretation of ICP slow waves may have a potential clinical value but user-friendly methods manageable in routine neurointensive care need to be developed and validated.

\section{Per Enblad}

Uppsala, Sweden

Publisher's note Springer Nature remains neutral with regard to jurisdictional claims in published maps and institutional affiliations. 